$P<0.01 / R=0.29 ; P=0.03)$. There was positive correlations between Fibromyalgia syndrome (FIRST scale) and lumbar VAS $(r=0.42 ; p=0.01)$ and root VAS $(R=$ $0.29 ; \mathrm{P}<0.01)$.

Conclusion: The results of this study showed that anxiety, depression and fibromyalgia syndrome are not only frequent with patient suffering from chronic LBP but they are also correlated to the pain intensity. As a consequence, assessment of and intervention in anxiety, depression symptoms and fibromyalgia syndrome is crucial in the provision of pain-relief nursing treatment in patients with low back pain.

Disclosure of Interests: None declared

DOI: 10.1136/annrheumdis-2021-eular.4086

\section{POS1288 \\ TUBERCULOUS SPONDYLODISCITIS IN ELDERLY: EPIDEMIOLOGY, CLINICAL, FEATURES, TREATMENT AND OUTCOMES}

M. Mrabet ${ }^{1}$, S. Boussaid ${ }^{1}$, S. Jemmali ${ }^{1}$, H. Sahli ${ }^{1}$, H. Ajlani ${ }^{1}$, E. Cheour ${ }^{1}$, S. Rekik ${ }^{1}$, M. Elleuch ${ }^{1} .{ }^{1}$ Rabta Hospital, Rheumatology, Tunis, Tunisia

Background: Tuberculosis is still endemic all over the world. The incidence of tuberculous spondylodiscitis (TS) is steadily increasing. Clinical features and outcomes of this affection are various and depending on various parameters, including age.

Objectives: Our objective was to explore the differences in presentation and the results of further investigations and the prognosis of TS between young and elderly subjects.

Methods: We conduct a retrospective and descriptive study in a single rheumatology department. Data were collected from files of patients hospitalized in the past 20 years $(2000-2020)$ who have been diagnosed with TS. We carried out a comparative study concerning the clinical biological, imaging features and outcomes between young subjects and subjects aged over 65 years.

Results: Fifty-two cases of TS were collected (37F/15M). The mean age of the population was 55.21 years \pm 17.79 [19-91]. Thirty-three patients $(69.2 \%)$ were classified as young versus 16 elderly patients (30.8\%), with female predominance in both groups $(69.4 \%$ and $75 \%$ respectively, $p=0.57)$. Young subjects was more frequently vaccinated (88.9\%) by Bacillus Calmette-Guérin (BCG) $(p<0.001)$. A delayed diagnosis was noted in both groups $(p=0.24)$. Lumbar spine involvement was the most common $(57.7 \%)$. In the two age ranges, the onset of the disease was progressive $(p=0.22)$, characterized by segmental spine stiffness ( $p=0.57)$ and lumbar pain with general signs $(p=0.27)$, such as: impaired general condition, fever, night sweats and weight loss. Biological inflammatory syndrome and normochrome normocytic anemia were encountered in both cases $(p=0.08$ and $p=0.2$, respectively). Standard X-rays and Computed tomography were more performed in young subjects $(94.4 \%$ and $69.4 \%$, respectively; $p<0.001)$, unlike magnetic resonance imaging which was more common in elderly subjects but with no statistically significant difference $(p=0.22)$. Disc pinch, erosion of vertebral plateaus and vertebral collapse were the major signs $(82.7 \%, 65.4 \%$ and $67.3 \%$, respectively). Clinical, biological and imaging arguments contributed to positive diagnosis in both groups $(p=0.24)$. Common medical treatment was anti-tuberculosis: Isoniazid $(H)$, Rifampicin $(R)$, Pyrazinamide $(Z)$, Ethambutol $(E)$ and physical treatment such as immobilization witch was more common in the eldery $(56.3 \%, p=0.16)$. The evolution of the disease was characterized by a clear improvement of young subjects during the second week of treatment $(p<0.001)$. A more frequent clinical improvement in older subjects was during the fourth week but with no statistically significant difference $(p=0.13)$. The occurrence of immediate complications was more frequent in the elderly $(p=0.23)$ with a predominance for drug complications (56.3\%) such as: hepatic cytolysis (12.5\%), hyperuricemia (18.8\%) and major intolerance to anti-tuberculosis (18.8\%).

Conclusion: TS is a frequent condition that needs to be treated rapidly. the clinical presentation of TS in the elderly is less noisy which leads to more frequent complications and mortality.

Disclosure of Interests: None declared

DOI: 10.1136/annrheumdis-2021-eular.4129

\section{POS1289 CHRONIC LOW BACK PAIN: THERAPEUTIC STRATEGIES AND CLINICAL OUTCOMES IN A MILITARY POPULATION}

R. Dhahri ${ }^{1}$, A. Dghaies ${ }^{1}$, M. Slouma ${ }^{1}$, L. Metoui ${ }^{1}$, I. Gharsallah $^{1}$, I. Dorgham ${ }^{2}$, Y. Mallat ${ }^{2}$, R. Ayari ${ }^{2}$, K. Amri ${ }^{2} .{ }^{1}$ Military Hospital of Tunis, Rheumatology, Tunis, Tunisia; ${ }^{2}$ Military Hospital of Tunis, Orthopedics, Tunis, Tunisia

Background: Low back pain is an extremely common patient complaint. Most cases resolve quickly after the acute episode. However, a significant number of patients develop chronic low back pain; a persistent disabling condition. Patients suffer from unremitting pain and often become functionally impaired.

Objectives: The aim of this study is to describe the characteristics of chronic LBP, physical examination abnormalities, treatment strategies and the impact of LBP on the professional life of the patients.
Methods: It was a an analytical cross-sectional study including 50 patients with at least three months of LBP, in the department of rheumatology and orthopedics at the Military Hospital of Tunis between January 1st and March 31, 2020. All patients had a standardized investigation and clinical assessment.

Results: The study included $80 \%$ of active military serving members and $20 \%$ of administrative officers. The mean age of the patients was $41.9 \pm 8.4$ years and the sex ratio was 4.5 . Four patients were suffering from diabetes; two patients were suffering from high blood pressure. All the patients were suffering from chronic LBP lasting for an average of 66.4 months. LBP was associated with radicular pain in $78 \%$ of the cases. It was a unilateral radicular pain in $72 \%$ of the cases and bilateral in $28 \%$ of the cases. The main triggering factors were: carrying heavy loads in $98 \%$ of the cases, standing or sitting for long periods in $90 \%$ and $76 \%$ of the cases, tremors in $74 \%$ of the cases. Neuropathic pain was found in $26 \%$ of patients. Physical examination showed paravertebral muscle tenderness in $66 \%$ of the cases and slack abdominal muscles in $56 \%$ of the cases Assessement of range of flexion of the lumbar spine showed: fingertip to floor test was $18 \pm 12.2 \mathrm{~cm}[054 \mathrm{~cm}]$, schober test was $+3.8 \pm 1.2 \mathrm{~cm}[16 \mathrm{~cm}]$. The extension of lumbar spine was painful in $80 \%$ of the cases. A trigger point was found in $28 \%$ of the cases. Lasegue sign was positive in $18 \%$ of the cases. Leri's test was positive in $8 \%$ of the cases. Required treatments during the last episode of LBP were: Paracetamol $(62 \%)$, nonsteroidal antiinflammatory drugs $(26 \%)$, tramado $(4 \%)$, myorelaxant (4\%) and pregabalin (2\%). Half of the patients needed functional rehabilitation. Forty percent of the patients reported improvement; $46 \%$ of them reported improvement then recurrence of the pain, $8 \%$ of them reported no improvement and $4 \%$ reported worsening of the symptoms. Thirty six percent of the patients needed an average of 21 days of leave and $35 \%$ of them needed exemption from work for LBP problems. One patient needed an outplacement from his original work and one patient needed an early retirement.

Conclusion: Chronic low back pain can cause significant functional disability and commonly becomes frustrating for both patients and physicians to cope with and treat. There is still no consensus on the best way to manage chronic low back pain, and clinical guidelines are scarce. A combination of pharmacological agents and non-pharmacological methods is the most appropriate therapeutic regimen.

Disclosure of Interests: None declared

DOI: 10.1136/annrheumdis-2021-eular.4149

\section{\begin{tabular}{|l|l}
\hline POS1290 CYTOKINE BIOMARKERS IN COMMON LOW BACK \\
\hline
\end{tabular} PAIN}

R. Dhahri ${ }^{1}$, A. Dghaies ${ }^{1}$, M. Slouma ${ }^{1}$, L. Metoui ${ }^{1}$, I. Gharsallah ${ }^{1}$, I. Dorgham ${ }^{2}$, R. Ayari ${ }^{2}$, Y. Mallat ${ }^{2}$, K. Amri ${ }^{2}$, A. Tezeghdenti ${ }^{3}$, W. Dkhili ${ }^{3}$, R. Kochkar ${ }^{3}$, E. Ghazouani ${ }^{3} .{ }^{1}$ Military Hospital of Tunis, Rheumatology, Tunis, Tunisia; ${ }^{2}$ Military Hospital of Tunis, Orthopedics, Tunis, Tunisia; ${ }^{3}$ The Military Hospital of Tunis, Immunology, Tunis, Tunisia

Background: Common low back pain (LBP) is a common health problem affecting 50 to $80 \%$ of working age adults. It is one of the common and costly health problems in Tunisia. Actually, the role of the immune response and inflammatory cytokines in the pathogenesis of chronic pain has been of growing interest.

Objectives: The aim of this study was to assess whether pro and anti-inflammatory cytokines could be detected in serum in patients with LBP compared with healthy subjects and whether they could be related to pain severity and to clinical findings. Methods: It was a an analytical cross-sectional study including 50 patients with at least three months of LBP, in the department of rheumatology, orthopedics and immunology at the Military Hospital of Tunis between January 1st and March 31, 2020. All patients had a standardized clinical assessment.

Levels of serum cytokines IL-6, IL-8, IL-1 $\beta$ and TNF- $\alpha$, were measured using the chimiluminescence technique. Serum concentration of IL-10 was assayed by the enzyme-linked immunosorbent assay technique (ELISA). The normal levels of cytokines were determined in 50 healthy controls.

Results: The mean age of the patients was $41.9 \pm 8.4$ years and the sex ratio was 4.5. LBP duration was 66.4 months. The mean lumbar visual analog scale (VAS) was $4.5 \pm 1.9$, and the root VAS was $2.6 \pm 2.5$. Neuropathic pain was found in $26 \%$ of patients. The average BMI was $27 \pm 3.7 \mathrm{~kg} / \mathrm{m}^{2}$. Only serum level of IL-8 was significantly higher in subjects with LBP compared to healthy controls ( $p$ $<10-3)$. IL-1 $\beta$ was indetectable in both patients and controls. Positive correlations were found between IL-8 levels and anxiety/functional scores $(r=0.3 ; p=0.02 /$ $r=0.3 ; p=0.04$ ). IL- 6 was positively correlated with BMI, and negatively correlated with the Schober test. No correlations were found between serum levels of IL-6, IL-8, IL-10, TNF- $\alpha$ and pain intensity (VAS), neuropathic pain (DN4), fibromyalgia (FIRST), depression (HAD) and various radiological data.

Conclusion: Interleukin-8 is a biomarker of common low back pain and correlate with anxiety and functional disability. These results suggest that IL-8 may be a therapeutic target to reduce chronic back pain and reduce the social and profession impact.

Disclosure of Interests: None declared

DOI: 10.1136/annrheumdis-2021-eular.4205 